\title{
Erratum: Asymmetric nuclear matter in a parity doublet model with hidden local symmetry [Phys. Rev. C 92, 025201 (2015)]
}

Yuichi Motohiro, Youngman Kim, and Masayasu Harada

(Received 6 January 2017; published 15 May 2017)

DOI: 10.1103/PhysRevC.95.059903

We found some numerical errors in our computation. In addition, we changed the input of the pion decay constant from 93 to 92.3 MeV. From these modifications, our numerical results in Tables I, II, IV-VI, and Figs. 1-5 should be corrected. Furthermore, we correct a typographical error in Eq. (2.12). However, our findings shown in the abstract are intact: E.g., our model reproduces the properties of normal nuclear matter (binding energy, saturation density, incompressibility, and symmetry energy).

Equation (2.12) should be read as

$$
M=\xi_{L}^{\dagger} \sigma \xi_{R}=\sigma \xi_{L}^{\dagger} \xi_{R}=\sigma U .
$$

Below we show corrected tables and figures together with corrections to the main text if any.

TABLE I. Determined model parameters for given $m_{0}$. Here $m_{\omega}=783, m_{\rho}=776 \mathrm{MeV}$, and $\bar{m} \epsilon=m_{\pi}^{2} f_{\pi}$.

\begin{tabular}{lcccrr}
\hline \hline$m_{0}(\mathrm{MeV})$ & 500 & 600 & 700 & 800 & 900 \\
\hline$g_{1}$ & 9.03 & 8.49 & 7.82 & 7.00 & 5.97 \\
$g_{2}$ & 15.49 & 14.95 & 14.28 & 13.45 & 12.43 \\
$g_{\omega N N}$ & 6.75 & 6.60 & 6.22 & 5.34 & 3.49 \\
$g_{\rho N N}$ & 8.19 & 8.20 & 8.24 & 8.32 & 8.43 \\
$\bar{\mu}^{2} / f_{\pi}^{2}$ & 73.5 & 50.7 & 30.8 & 14.9 & 1.74 \\
$\lambda$ & 139 & 95.9 & 58.8 & 29.4 & 5.00 \\
$\lambda_{6} f_{\pi}^{2}$ & 62.9 & 42.9 & 25.7 & 12.2 & 0.952 \\
\hline \hline
\end{tabular}

TABLE II. Physical inputs in vacuum (MeV).

\begin{tabular}{cccccc}
\hline \hline$m_{+}$ & $m_{-}$ & $m_{\omega}$ & $m_{\rho}$ & $f_{\pi}$ & $m_{\pi}$ \\
\hline 939 & 1535 & 783 & 776 & 92.3 & 140 \\
\hline \hline
\end{tabular}

TABLE IV. Predicted values of the slope parameter for $m_{0}=$ 500-900 MeV.

\begin{tabular}{lc}
\hline \hline$m_{0}(\mathrm{MeV})$ & $L(\mathrm{MeV})$ \\
\hline 900 & 80.1 \\
800 & 81.1 \\
700 & 81.8 \\
600 & 82.2 \\
500 & 82.4 \\
\hline \hline
\end{tabular}

TABLE V. Critical values of the baryon chemical potential $\mu_{B}^{c}$ at $T=0$ and $\mu_{I}=0 \mathrm{MeV}$.

\begin{tabular}{lcccc}
\hline \hline$m_{0}(\mathrm{MeV})$ & $\mu_{B \lg }^{c}(\mathrm{MeV})$ & $\rho_{B \lg }^{c}\left(\mathrm{fm}^{-3}\right)$ & $\mu_{B_{\chi}^{c}}^{c}(\mathrm{MeV})$ & $\rho_{B \chi}^{c}\left(\mathrm{fm}^{-3}\right)$ \\
\hline 900 & 923 & 0.16 & 1532 & 2.97 \\
800 & 923 & 0.16 & 1580 & 1.77 \\
700 & 923 & 0.16 & 1412 & 1.19 \\
600 & 923 & 0.16 & 1220 & 0.89 \\
500 & 923 & 0.16 & 1055 & 0.72 \\
\hline \hline
\end{tabular}

TABLE VI. Critical values of the baryon chemical potential and density at $T=0$ and $\mu_{I}=100 \mathrm{MeV}$.

\begin{tabular}{lcccc}
\hline \hline$m_{0}(\mathrm{MeV})$ & $\mu_{B \lg }^{c}(\mathrm{MeV})$ & $\rho_{B \lg }^{c}\left(\mathrm{fm}^{-3}\right)$ & $\mu_{B_{\chi}^{c}}^{c}(\mathrm{MeV})$ & $\rho_{B_{\chi}^{c}}^{c}\left(\mathrm{fm}^{-3}\right)$ \\
\hline 900 & 892 & 0.0 & 1530 & 2.97 \\
800 & 890 & 0.0 & 1578 & 1.77 \\
700 & 889 & 0.0 & 1408 & 1.19 \\
600 & 889 & 0.0 & 1214 & 0.88 \\
500 & 889 & 0.0 & 1045 & 0.70 \\
\hline \hline
\end{tabular}


Associated with the modification in Fig. 2, " $\mu_{B} \sim 1300 \mathrm{MeV"}$ " that appears in the seventh paragraph of Sec. IV should read " $\mu_{B} \sim 1050 \mathrm{MeV}$."

Associated with the modification in Fig. 4, in the second paragraph of Sec. V, the text "(blue solid line)" and "(red solid line)" should be deleted, and "the green dashed line" should read as "the dashed line."

Associated with the modification in Fig. 5, the last sentence of the fifth paragraph in Sec. V, "As expected, the nature of the liquid-gas transitions does not change, while the first-order chiral phase transition becomes second order." should be read as "As expected, the nature of the liquid-gas transitions does not change, whereas the order of the chiral phase transition is changed."

Associated with the modification in Table V, the values in the first and second sentences of the seventh paragraph in Sec. V are changed as follows: " $17 \rho_{0}$ " to $18.5 \rho_{0}$ and " $3 \rho_{0}$ " to $4.5 \rho_{0}$.
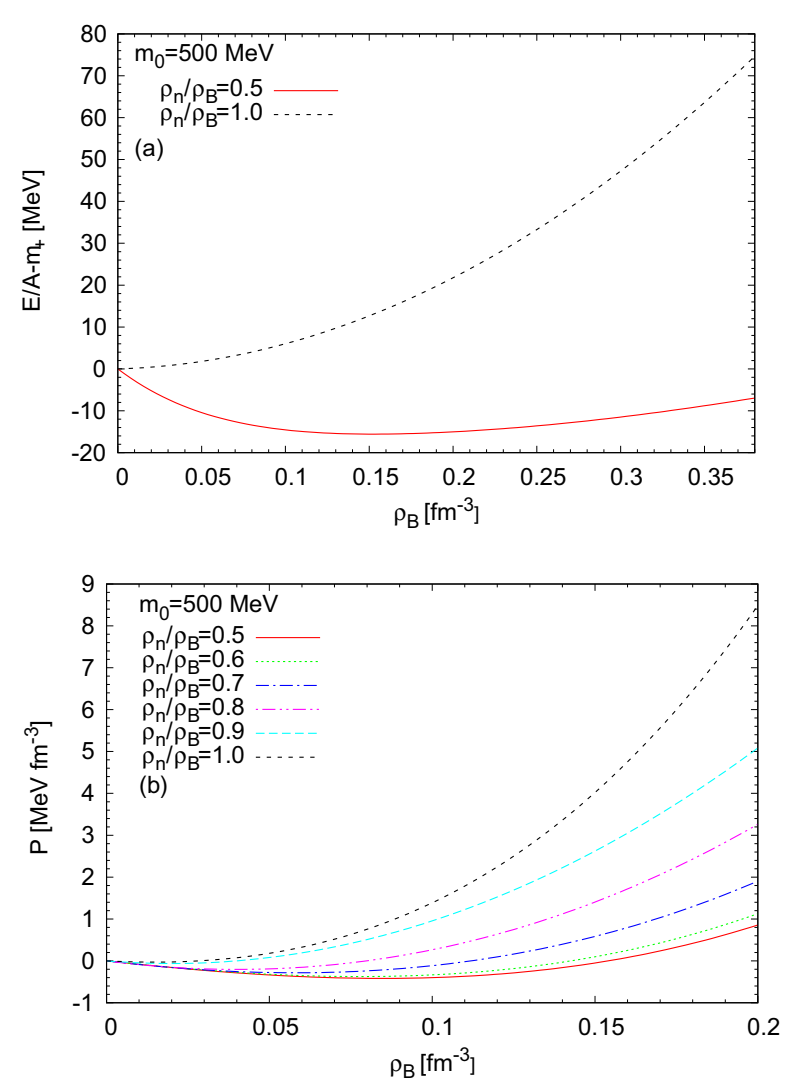

FIG. 1. (a) Density dependence of the binding energy. (b) The pressure for $m_{0}=500 \mathrm{MeV} . \rho_{B}$ is the baryon number density, and $\rho_{n}$ is the neutron number density: $\rho_{n} / \rho_{B}=0.5$ implies symmetric nuclear matter, and $\rho_{n} / \rho_{B}=1$ implies pure neutron matter.
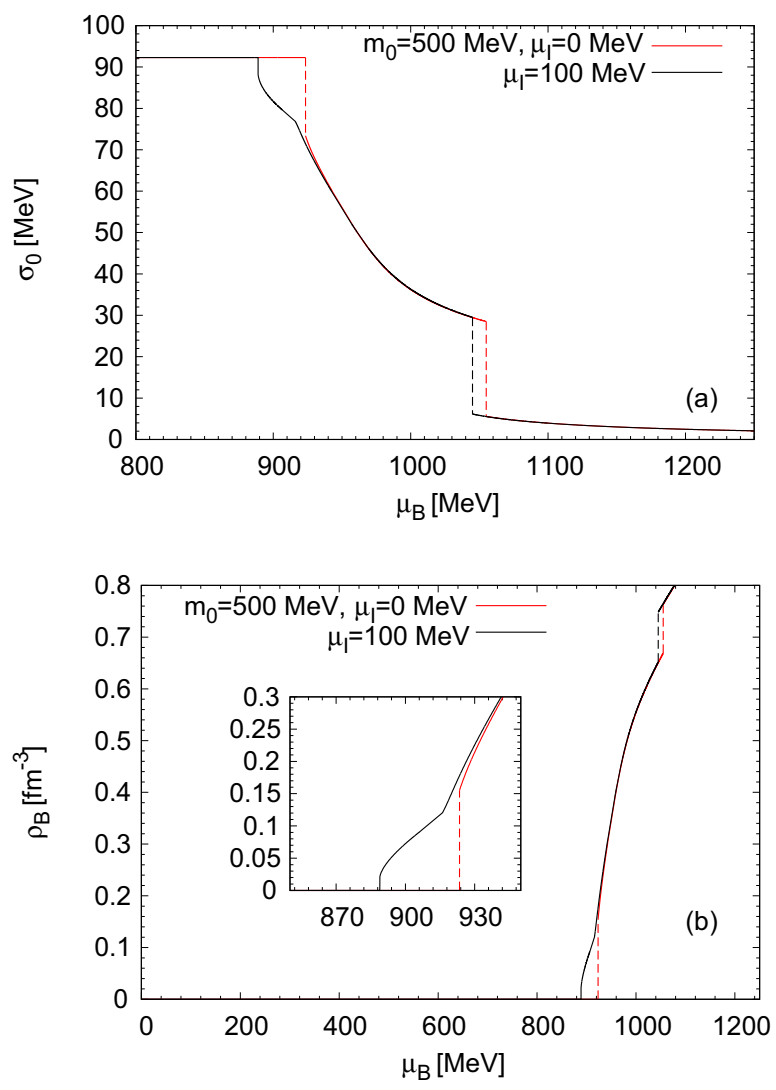

FIG. 2. (a) Baryon number chemical potential dependence of $\sigma_{0}$. (b) Chemical potential dependence of $\rho_{B}$ for $m_{0}=500$ and $\mu_{I}=0 \mathrm{MeV}$.

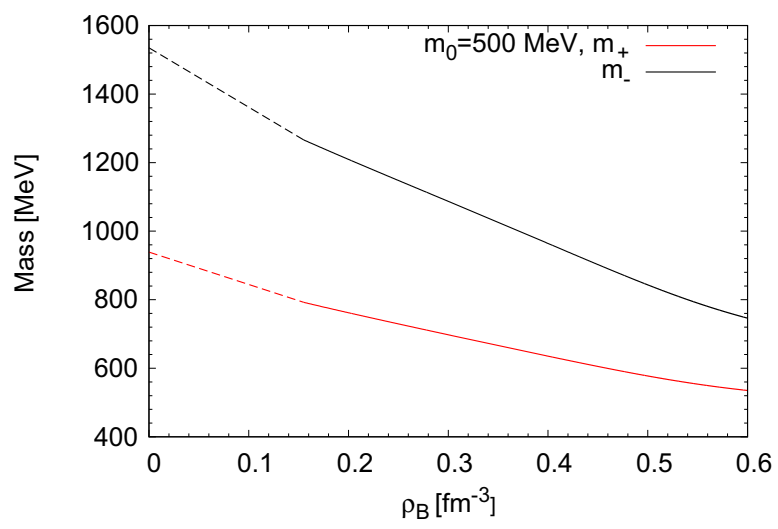

FIG. 3. Density dependence of the effective nucleon masses for $m_{0}=500 \mathrm{MeV}$ at $\mu_{I}=0 \mathrm{MeV}$. 

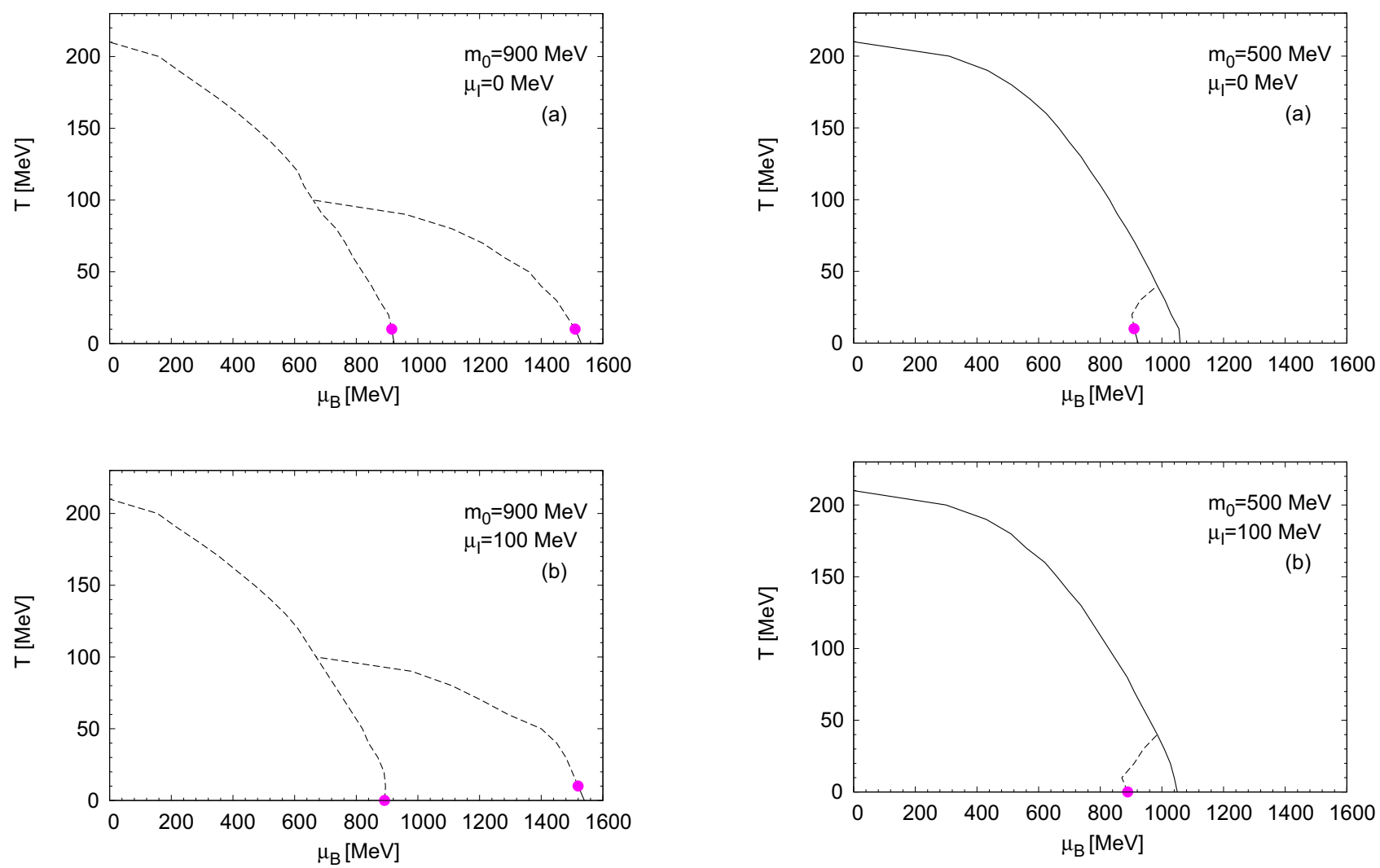

FIG. 4. Phase diagrams for $m_{0}=900 \mathrm{MeV}$ at (a) $\mu_{I}=0 \mathrm{MeV}$ and (b) $\mu_{I}=100 \mathrm{MeV}$. The solid line is for the first-order phase transition, the dashed line is for the crossover, and the point is for the critical point (second order).

FIG. 5. Phase diagrams for $m_{0}=500 \mathrm{MeV}$. 